Cahiers « Mondes anciens »

MONDES

ANCIENS

Histoire et anthropologie des mondes anciens

$6 \mid 2015$

Mères grecques

\title{
Les Nymphes entre maternité et courotrophie dans les Hymnes homériques
}

Nymphs between Motherhood and Courotrophy in the Homeric Hymns

\section{Sébastien Dalmon}

\section{(2) OpenEdition}

Journals

Édition électronique

URL : http://journals.openedition.org/mondesanciens/1471

DOI : 10.4000/mondesanciens. 1471

ISSN : 2107-0199

Éditeur

UMR 8210 Anthropologie et Histoire des Mondes Antiques

Référence électronique

Sébastien Dalmon, «Les Nymphes entre maternité et courotrophie dans les Hymnes homériques », Cahiers « Mondes anciens » [En ligne], 6 | 2015, mis en ligne le 29 janvier 2015, consulté le 19 avril 2019 URL : http://journals.openedition.org/mondesanciens/1471; DOI : 10.4000/mondesanciens. 1471

Ce document a été généré automatiquement le 19 avril 2019

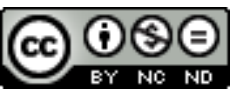

Les Cahiers «Mondes Anciens " sont mis à disposition selon les termes de la licence Creative Commons Attribution - Pas d'Utilisation Commerciale - Pas de Modification 4.0 International. 


\title{
Les Nymphes entre maternité et courotrophie dans les Hymnes homériques
}

\author{
Nymphs between Motherhood and Courotrophy in the Homeric Hymns
}

\author{
Sébastien Dalmon
}

1 Les Nymphes grecques sont souvent présentées comme des nourrices et des puissances courotrophes. Cette dimension a été soulignée dès l'article fondateur sur le culte des Nymphes de Floyd G. Ballentine (1904, p. 102-106) qui insiste sur le rôle de ces divinités dans les domaines de la naissance et du soin des enfants. Elle est rappelée dans des ouvrages plus récents, comme celui de Christiane Sourvinou-Inwood (2005, p. 106-108). Mais les Nymphes peuvent aussi être parfois des mères, et ce dès l'épopée homérique ; ainsi les mères Naïades de héros alliés des Troyens dans l'lliade (VI, 20-28; XIV, 442-445; XX, 384-385). La maternité est cependant un terme polysémique, désignant d'une part « une fonction reproductive ", «l'action de porter et mettre au monde un enfant » et d'autre part «la relation (matérielle et affective) tissée avec des enfants et inscrite dans un réseau de parenté » (Thébaud 2005, p. 9 ; Cova 2005, p. 190) ${ }^{1}$. Vinciane Pirenne-Delforge (2005) l'a bien montré, pour les déesses grecques, en distinguant par exemple la «capacité reproductive » des mères de la Théogonie hésiodique (Gaia, Rhéia et Déméter², qui n'en montrent pas moins une grande sollicitude pour leurs enfants) de la maternité sociale et symbolique de la parthenos Athéna à Élis (où elle porte l'épiclèse de mêtêr) ou à Athènes où elle élève l'autochtone Érichthonios, modèle des citoyens athéniens ${ }^{3}$.

Dans la deuxième définition précitée, on n'est pas très loin de la fonction de nourrice et de courotrophe, il suffit de retrancher la mention «et inscrite dans un réseau de parenté ». Cependant, la notion grecque de kourotrophos est plus large que celle de nourrice et « recouvre le long processus de la formation des êtres humains, depuis la conception jusqu'à l'âge où le koũ pos change de statut et devient un homme » (PirenneDelforge 2004, p. 173), même si, dans le vocabulaire des études iconographiques, elle sert 
à désigner une figure, généralement féminine, portant un enfant dans ses bras ou sur ses genoux (Hadzisteliou-Price, 1978).

Dans la préface d'un ouvrage collectif consacré à La puissance maternelle en Méditerranée, Yvonne Knibiehler (2008), qui distingue puissance biologique, puissance sociale et puissance affective des mères, propose, pour cette dernière, « de revisiter attentivement le recours aux nourrices. Il faut y voir, assurément, une intention de séparer l'enfant de sa mère, pour réduire l'influence de celle-ci. Mais en pratique, dans quelle mesure la mère se dessaisit-elle du bébé ? Quels étaient les rapports entre la mère et la nourrice, entre la nourrice et le nourrisson, entre la mère et le nourrisson?» (p.14). On peut se poser ce genre de questions pour le monde grec. La langue grecque connait deux termes pour le mot «nourrice » : $\dot{\eta} \tau \imath \theta \dot{\eta} v \eta$ ou $\dot{\eta} \tau \rho o \varphi o ́ \varsigma$, renvoyant tous deux à l'acte de nourrir et de favoriser la croissance, même si le premier terme semble impliquer l'allaitement (Demont 1978, Pirenne-Delforge 2010). Cependant la tâche de la nourrice grecque n'est généralement pas d'allaiter l'enfant ; dans la tradition épique, il s'agit d'une fille vierge ou d'une femme ménopausée qui ne peuvent donc avoir de lait. L'allaitement étant une prérogative maternelle, il n'y a donc pas concurrence entre le rôle de mère et celui de nourrice, qui est généralement sa subalterne, une esclave le plus souvent, qui chérit l'enfant, veille à son sevrage et à son apprentissage de l'autonomie. Les Nymphes, divinités secondaires au service de divinités plus importantes, sont souvent les nourrices de dieux (Zeus, Dionysos) ou de héros (Énée); dans ces récits mythologiques, l'allaitement est souvent assuré par un animal, comme la chèvre Amalthée pour Zeus (Vilatte, 1991, p. 7-13).

4 Les Nymphes oscillent dans nos sources entre maternité et courotrophie (ou plutôt paidotrophie, car on s'intéressera ici plus au soin accordé aux jeunes enfants qu'aux jeunes gens). L'étude sera limitée aux hymnes homériques ${ }^{4}$, qui offrent un bon condensé des différents rôles que peuvent jouer les Nymphes entre maternité biologique, maternité sociale et fonction de nourrice, cette dernière semblant y être attestée pour la première fois au sein de la littérature grecque. Ces poèmes présentent une certaine unité dans leur forme, car ils relèvent, comme l'Iliade, l'odyssée ou la Théogonie, du genre épique. Ils se structurent de manière tripartite entre une invocation à la divinité concernée par l'hymne (évoquée généralement à la troisième personne), une partie descriptive et narrative faisant l'éloge du dieu et se concentrant souvent sur un épisode de sa biographie divine (ayant pour sujet ses timai) et une adresse finale directe à la divinité (semblant ouvrir dans certains cas à un autre poème). Ces hymnes peuvent être datés pour la plupart de l'époque archaïque, même si certains relèvent de l'époque classique, voire hellénistique ${ }^{5}$. Les Nymphes ne sont pas le sujet en tant que telles de ces hymnes, mais elles apparaissent dans certains d'entre eux. Il s'agira ainsi de voir ce que l'analyse de ces textes peut nous apprendre, au sein du système polythéiste grec, sur le mode d'action et les fonctions des Nymphes dans les domaines de la maternité et de la courotrophie.

5 Les Nymphes mères et nourrices sont surtout présentes, selon des modalités parfois fort différentes, dans l'Hymne homérique à Hermès (où prédomine la figure de la Nymphe Maïa) et l'Hymne homérique à Aphrodite. Mais elles occupent également une place non négligeable dans le deuxième Hymne homérique à Dionysos et dans l' Hymne homérique à Pan, généralement considérés comme plus tardifs. 


\section{La Nymphe Maïa dans l'Hymne homérique à Hermès : mère et nourrice à la fois}

6 Les différents éditeurs du premier Hymne homérique à Hermès s'accordent à le dater du vi ${ }^{\mathrm{e}}$ siècle av. J.-C. (Humbert 1976, p. 115 ; Evelyn White 1967, p. xxxviii) ${ }^{6}$. Ce poème chante la naissance du dieu dans une grotte du Cyllène et les exploits qu'il accomplit entre deux levers de soleil (invention de la lyre, rapt des vaches d'Apollon, invention du feu) afin d'obtenir de son père Zeus, le roi des dieux, sa part de timê et son intégration à l'ordre olympien (Leduc 2005, p. 141 ; Jaillard 2007). Le poème évoque dès ses premières lignes la figure de la mère d'Hermès, la Nymphe Maïa :

Muse, chante un hymne à Hermès, fils (vióv) de Zeus et de Maïa, roi du Cyllène et de l'Arcadie abondante en moutons, messager bienfaisant des Immortels, à celui

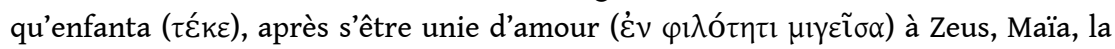

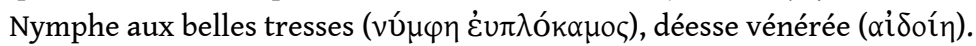

Fuyant la société des Dieux bienheureux, elle demeurait dans un antre plein d'ombre; c'est là que le Cronide venait, en pleine nuit, s'unir à la Nymphe aux

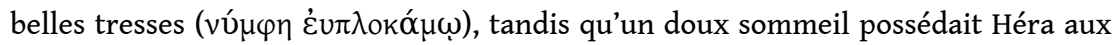
bras blancs - à l'insu des Dieux immortels et des hommes mortels. Mais lorsque le dessein du grand Zeus fut près de s'accomplir - elle voyait alors la dixième Lune se fixer au ciel - et qu'il fit paraître au jour, dans leur achèvement, ses glorieuses

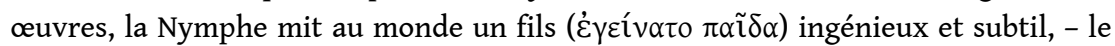
Brigand, le Ravisseur de bœufs, l'Introducteur des songes, le Guetteur nocturne, le Rôdeur de portes - qui devait bientôt manifester parmi les Dieux immortels des actions éclatantes (Hymne Homérique à Hermès I, 1-16) ${ }^{7}$.

Dès le premier vers de l'hymne, Hermès est donné comme le fils (vióv) de la Nymphe Maïa, même si celle-ci n'est pas explicitement désignée comme «mère ». Il est ensuite considéré comme «enfant » ( $\pi \alpha \tilde{i} \delta \alpha)$ de la Nymphe au vers 13. Le vocabulaire utilisé est

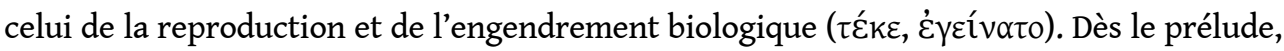
Hermès est rattaché à son lieu de naissance, l'Arcadie, et plus spécifiquement au mont

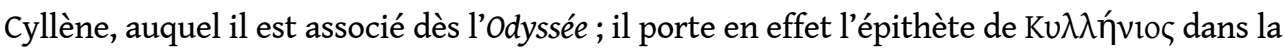
seconde nekuia, où il intervient dans sa fonction de psychopompe pour escorter les Prétendants défunts dans l'Hadès (Homère, Odyssée, XXIV, 1). Hermès est présenté dans l'hymne comme le messager des dieux, ce qui participe, comme l'a montré Laurence Kahn, de son mode d'action comme médiateur spatial et médiateur de l'échange, marqué par l'équivoque de la mêtis, l'intelligence rusée, façon d'indiquer les «ambiguïtés de la communication » (Kahn 1978, notamment p. 177-180).

8 Sa mère apparait d'abord dans un contexte érotique comme s'étant unie d'amour ( $\dot{\varepsilon} v$ $\varphi \imath \lambda o ́ \tau \eta \tau \imath \mu ı ⿻ \tilde{\tau} \sigma \alpha)$ à Zeus. Cette union ressemble à celle des Nymphes avec les Silènes ou avec Hermès lui-même dans l'Hymne homérique à Aphrodite I (262-263), où il est dit également qu'ils «s'unissent amoureusement au fond des grottes charmantes " ( $\mu$ í $\sigma \gamma o v \tau^{\prime}$

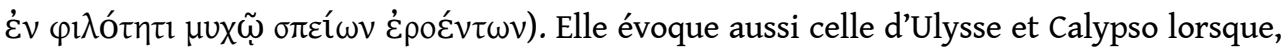
" au profond de la grotte, ils rentrèrent pour rester dans les bras l'un de l'autre à s'aimer » (Homère, Odyssée, V, 226-227). C'est une union illégitime, la numphê Maïa s'opposant à l'épouse légitime et accomplie Héra, qui dort à poings fermés, ignorante de son infortune conjugale.

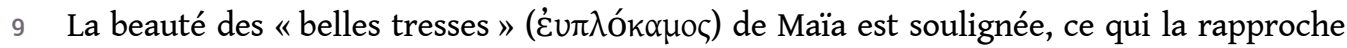

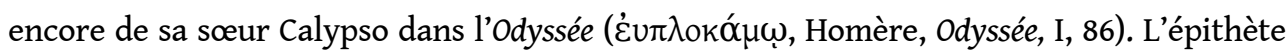


est répétée dans le vers 7, ainsi que dans le second Hymne homérique à Hermès (au même vers 7), et apparaît donc, semble-t-il, comme une expression formulaire qualifiant Maïa.

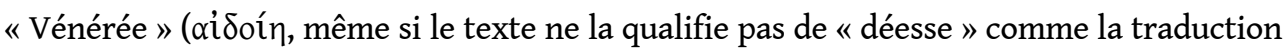
de Jean Humbert) autant que sa sœur Calypso qui est qualifiée de «Nymphe auguste »

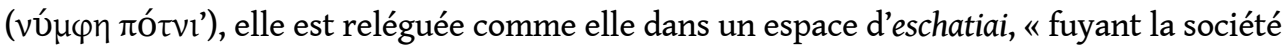
des Dieux bienheureux » et habitant " un antre plein d'ombre », ce qui n'est pas sans un caractère quelque peu inquiétant et rappelle par trop la cruelle Echidna de la Théogonie d'Hésiode, vivant dans une caverne «bien loin des dieux immortels et des humains mortels » (v. 302). Mais cet antre est surtout le cadre des amours de la Nymphe et du dieu souverain, même si cette liaison a lieu dans la discrétion la plus absolue ("à l'insu des dieux immortels et des hommes mortels »), pour ne pas dire la dissimulation. Les replis de la grotte obscure sont également le lieu de conception et de naissance d'Hermès, un lieu qui est donc lié à la sexualité, mais aussi à la procréation. On pourrait presque y voir une métaphore du sexe féminin ${ }^{8}$, non celui de la Terre (Hermès n'est pas un gegênês!), mais bien celui de Maïa. Rappelons au reste que le mot numphê avait quelque chose à voir, en Grèce, avec les organes génitaux féminins, désignant tantôt le clitoris, tantôt les grandes ou petites lèvres (Winkler 2005, p. 341-342), qui «cachent » l'entrée de la matrice. Les Nymphes semblent donc avoir quelque affinité avec les dissimulations, ce que l'on pouvait déjà soupçonner, dans l'odyssée, avec Calypso, «Celle qui cache » (nom dérivé du verbe kaluptein, « cacher, couvrir, envelopper »)... En fait, on n'en attendrait pas moins de nièces du rusé Prométhée, qui cache sous la panse du premier bœuf sacrifié toutes les parties comestibles de l'animal, et sous l'appétissante graisse blanche les os inutiles ${ }^{9} .$. Le fils qui naît de cette union, « fils ingénieux et subtil, - le Brigand, le Ravisseur de bœufs, l'Introducteur des songes, le Guetteur nocturne, le Rôdeur de portes », est digne d'une telle hérédité.

Après avoir inventé la lyre, le jeune Hermès en joue tout en chantant les amours de ses parents « Zeus le Cronide et Maïa aux belles sandales ( $\left.\kappa \alpha \lambda \lambda_{\imath} \pi \varepsilon \delta^{\delta} \backslash \lambda \circ v\right)$ » qui s'unissent dans la philotês (Hymne Homérique à Hermès I, 54-62). Cependant, là non plus, Maïa n'est pas qualifiée de mère. Compte ici, précisément, sa relation à Zeus, non à son fils.

On retrouve le vocabulaire de l'engendrement, et même de l'accouchement (utilisation du verbe $\lambda$ oxع́́ $(\omega)$, quand Apollon atteint la grotte de Maïa dans la quête de ses bœufs :

À ces mots l'Archer Apollon, fils de Zeus, s'élança et atteignit le mont Cyllène que la

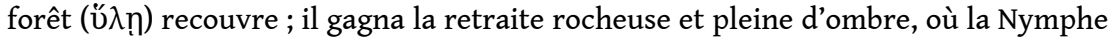

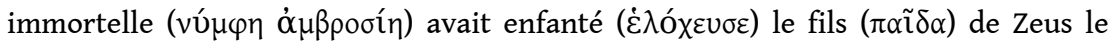
Cronide (Hymne Homérique à Hermès I, 227-230).

Maïa, à la différence des Nymphes de l'Hymne homérique à Aphrodite, est ici clairement

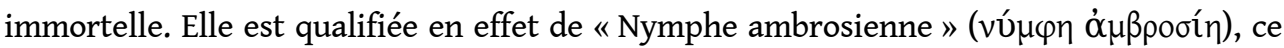
qui souligne son affinité avec la nourriture d'immortalité. Elle ressemble en cela à sa sœur Calypso, proche des sources de l'ambroisie transportée par les colombes de Zeus (Ballabriga 1986, p. 90-95), et qui se nourrit de ce mets ${ }^{10}$ et de nectar tout en proposant l'immortalité à Ulysse (Homère, Odyssée, V, 196-210). Ce qualificatif d'« ambrosienne " suggère peut-être que la mère d'Hermès nourrit son fils de cet aliment divin, même si l'enfant ne grandit pas de façon accélérée comme Apollon nourri de nectar et d'ambroisie par Thémis dans l'Hymne homérique à Apollon I, 123-129.

L'arrivée d'Apollon, justement, devant la grotte de Maïa, donne lieu à un développement intéressant : 
Loin de les méconnaître, le fils de Zeus et de Léto reconnut la belle Nymphe de la montagne ( $v u ́ \mu \varphi \eta v \tau^{\prime}$ oủ $\rho \varepsilon i ́ \eta v \pi \varepsilon \rho \kappa \alpha \lambda \lambda \varepsilon^{\prime} \alpha$ ) et son cher fils ( $\varphi$ ílov vióv), cet enfant

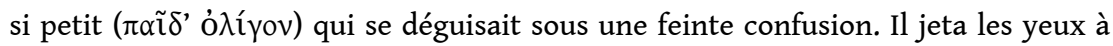

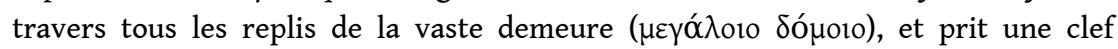
brillante pour ouvrir trois retraits pleins de nectar et d'ambroisie délectable. Il y avait là-dedans beaucoup d'or et d'argent, beaucoup de vêtements à la Nymphe -

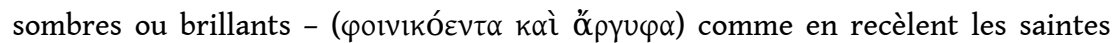

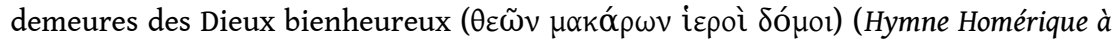
Hermès I, 243-251). probablement d'ambroisie qu'est nourri le nouveau-né, dont sa mère la Nymphe semble s'occuper avec sollicitude, le nourrissant, le langeant et lui donnant des bains. Il convient de souligner que le mot de mêtêr est explicitement employé pour la Nymphe. Maïa ne se contente donc pas d'être seulement une génitrice, elle prend soin de l'enfant et le sollicite comme le ferait une nourrice. Au reste, Maïa est également, dans d'autres sources, la nourrice d'Arcas, fils de Callisto et de Zeus (Borgeaud 1979, p. 49). Dans l'épopée, le nom même de " maïa » - qu'on pourrait traduire par " petite mère ", «bonne mère ", parfois « grand-mère » (Loraux 1991, p. 47) - est un qualificatif affectueux que la nourrice reçoit des enfants qu'elle a élevés (cf. Euryclée dans l'Odyssée, I, 435 ; II, 349 ; XIX, 500). La Nymphe Maïa semble donc apparaître à la fois comme une mère et comme une nourrice. Dans les Limiers de Sophocle, qui portent sur le même sujet que l'hymne (le vol des troupeaux d'Apollon), cette dernière fonction est plutôt remplie par la Nymphe éponyme de la montagne, Cyllène. montagnarde, habitat qui la rapproche de nombreuses autres Nymphes liées aux grottes et aux montagnes. L'hymne met aussi l'accent sur sa beauté et sa séduction, ainsi que sur sa capacité de dissimulation, qui pourrait être l'un des modes d'action privilégiés des Nymphes. Même si les premières références que nous avons analysées mettent plutôt l'accent sur la maternité biologique de Maïa, sa sollicitude maternelle est ensuite évoquée. La déesse prend soin elle-même de son fils Hermès, ce qui la rapproche des Nymphes nourrices, comme celles de l'Hymne homérique à Aphrodite.

\section{Les nourrices d'Énée dans l'Hymne homérique à Aphrodite}

L'Hymne homérique à Aphrodite paraît être à peu près contemporain de l'Hymne homérique à Déméter. Il remonte donc probablement à la fin du VII ${ }^{\mathrm{e}}$ siècle av. J.-C. ${ }^{11}$ Les Nymphes sont 
nommées à cinq reprises : les deux premières mentions sont le fait d'Anchise, qui croit avoir affaire à une Nymphe alors qu'il a devant lui Aphrodite déguisée en jeune mortelle pour le séduire (vers 97 et 98). La mention suivante évoque des numphai humaines au seuil du mariage (vers 119). Enfin, les deux dernières évoquent les Nymphes des arbres de l'Ida, nourrices d'Énée, fils d'Aphrodite et d'Anchise. C'est à elles que l'on va ici s'intéresser.

Aphrodite évoque dans un long discours le statut particulier des Nymphes de l'Ida à qui elle compte confier son enfant à naître. Elle ordonne à Anchise de cacher la vérité sur son aventure avec elle et de prétendre que son fils est né de l'une de ces Nymphes :

Sitôt qu'il verra la lumière du soleil, ce fils aura pour nourrices des Nymphes

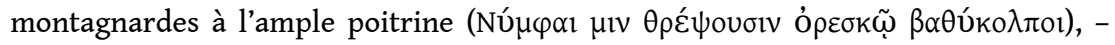

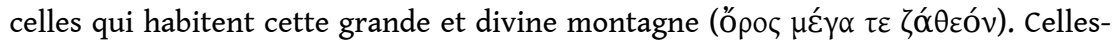

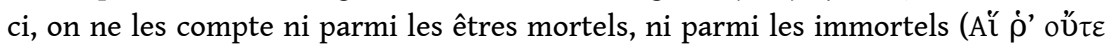

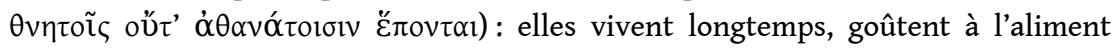

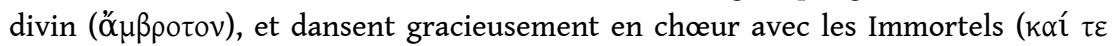

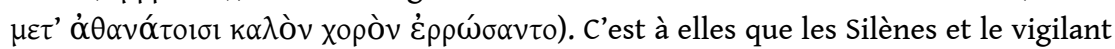
Argeiphontès s'unissent amoureusement au fond des grottes charmantes ( $\mu$ í $\sigma \gamma o v \tau$ '

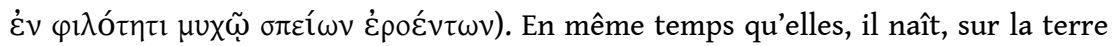

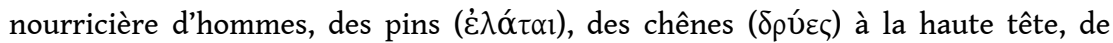
beaux arbres qui grandissent sur les hautes montagnes : ils se dressent, immenses, et on les appelle les bois sacrés ( $\tau \varepsilon \mu \varepsilon ́ v \eta)$ des Immortels. Jamais les mortels ne les abattent avec le fer ; mais quand arrive l'heure fatale de la mort, on les voit d'abord sécher sur le sol, ces beaux arbres; l'écorce dépérit tout autour du tronc, et les branches tombent : alors, en même temps, l'âme ( $\psi v x \grave{\eta})$ (des Nymphes) abandonne la lumière du Soleil. Ce sont elles qui garderont mon fils à leurs côtés et l'élèveront

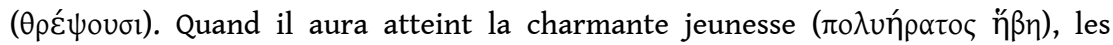
déesses $(\theta \varepsilon \alpha i ́)$ ramèneront ici ton fils $(\pi \alpha i \tilde{\delta} \alpha)$ et te le présenteront. Pour moi, je reviendrai dans sa cinquième année te voir avec l'enfant (viòv), afin de te remettre tout cela dans l'esprit: dès que tu verras de tes yeux ce jeune être florissant, tu te réjouiras à sa vue - car il ressemblera tout à fait à un dieu - et tu l'emmèneras aussitôt dans la venteuse Ilion (Hymne Homérique à Aphrodite I, 256-280).

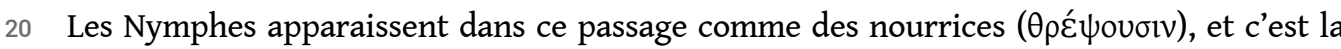
première occurrence, pour elles, dans les textes, de cette fonction, promise à une grande fortune poétique ${ }^{12}$. Ici, elles sont les nourrices d'un héros, qui est aussi un demi-dieu car né des amours d'une déesse et d'un mortel. L'enfant qui leur est confié est donc, comme elles, dans un statut intermédiaire (Clay 1989, p. 194), même s'il participe beaucoup plus de la nature humaine. Les Nymphes assurent une transition entre sa naissance sur l'olympe et son retour au monde des hommes, dans la ville de son père (Brillet-Dubois 2001, p. 253). Leur fonction de nourrice est renforcée par l'évocation de leur «ample poitrine » ( $\beta \alpha \theta$ v́колто) qui accompagne leur qualification de Nymphes montagnardes

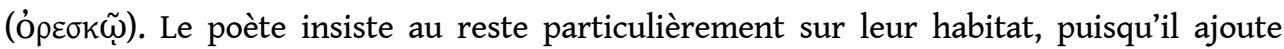

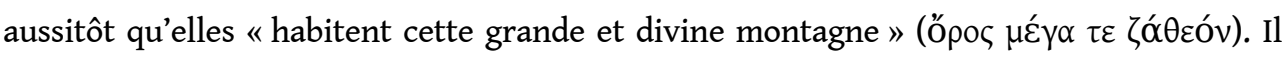
convient de noter que la montagne, par sa position médiane entre terre et ciel, représente souvent un espace de transition entre monde des dieux et monde des hommes, un lieu où le divin et l'humain se côtoient (Buxton 1996, p. 111).

21 Vient ensuite ce qui a retenu le plus l'attention des commentateurs de ce passage, à savoir l'évocation de la mortalité des Nymphes $^{13}$, qu'on ne trouve pas chez Homère (au contraire, Calypso offre même l'immortalité à Ulysse), et, chez Hésiode, uniquement dans un passage de localisation incertaine où une Naïade déclare qu'elle et ses sœurs vivent 9720 générations de mortels, sans partager, donc, l'immortalité des dieux (Hésiode, 
fragment 304 Merkelbach-West = Plutarque, Sur la disparition des oracles, 11, 415cd). C'est une façon de souligner leur statut intermédiaire, car « on ne les compte ni parmi les êtres

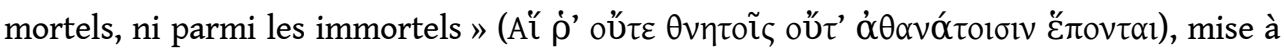
distance des deux groupes qui n'est pas exactement équivalente, même si elle s'en rapproche, de ce qui nous est dit de Calypso dans l'Odyssée, d'Echidna dans la Théogonie hésiodique et de Maïa dans l'Hymne homérique à Hermès. Car ces dernières, même si elles vivent « loin des dieux et des hommes ", n'en sont pas moins immortelles.

Ces Nymphes mènent une vie qui ressemble à celle des hommes de la race d'or chez Hésiode, vivant longtemps et se nourrissant des mêmes nourritures que les dieux,

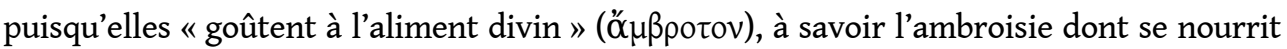
aussi Calypso dans l'Odyssée, et qui qualifie Maïa dans l'Hymne homérique à Hermès. Elles restent éternellement jeunes, belles et séduisantes. Jenny Strauss Clay souligne que cet excursus sur la condition des Nymphes est loin d'être une digression gratuite. Elle s'inscrit en effet dans l'ensemble du poème, complétant une série d'exemples donnés par Aphrodite à Anchise, commençant avec l'histoire de Ganymède, et se poursuivant avec celle de Tithon. Le blond Ganymède, enlevé par Zeus pour devenir l'échanson des dieux, devient immortel et reste éternellement jeune. C'est donc lui qui a le sort le plus enviable. Son parent Tithon a beaucoup moins de chance : il est également immortel, mais vieillit jusqu'à la décrépitude, enfermé dans le palais d'Éos. Le statut des Nymphes est l'exact inverse de celui de l'amant de l'Aurore, car elles restent jeunes tout en demeurant mortelles. Elles ont un statut divin dès l'origine, alors que le fils de Tros n'est qu'un homme divinisé. En contraste à la fois avec les nourrices de son fils, Ganymède et Tithon (qui appartiennent tous deux comme lui à la lignée royale troyenne), Anchise demeure un mortel sujet au vieillissement. Cette comparaison structurale des mortels aimés des dieux ${ }^{14}$, entre Ganymède (immortel, éternellement jeune), Tithon (immortel, sujet au vieillissement), les Nymphes de l'Ida (mortelles, mais restant jeunes) et Anchise (mortel, sujet au vieillissement) peut être complétée par celle des régimes alimentaires (nectar et ambroisie pour Ganymède ; blé et ambroisie pour Tithon; nourriture ambroisienne pour les Nymphes; blé simplement pour Anchise) et celle des lieux (dans l'olympe pour Ganymède; sur les bords d'Océan, au bout de la terre, pour Tithon; dans les espaces sauvages de l'Ida pour les Nymphes; à Troie pour Anchise ; cf. Segal 1974). Tithon et les Nymphes représentent en fait les deux points intermédiaires entre les polarités de la mortalité/immortalité et de la jeunesse/vieillesse, mais le modèle tithonien représente un échec de la médiation. Sa vieillesse éternelle est en effet un sort pire que la mort, et Aphrodite exploite son exemple pour réconcilier Anchise avec son statut de mortel (Clay 1989, p. 194-195). Les Nymphes, quant à elles, offrent un exemple de médiation réussie entre les hommes et les dieux : dans leur rôle de nourrices d'Énée, elles font en effet figures d'intermédiaires entre sa mère divine (qui leur donne l'enfant une fois né) et son père mortel (à qui elles le remettront).

Figures courotrophes, les Nymphes n'en sont pas moins érotisées. Elles peuvent s'unir avec des dieux comme Hermès ou avec les Silènes. Dans ces unions « au fond des grottes

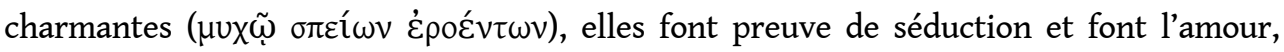
semble-t-il, sans violence, se «mélangeant dans la bonne entente» ( $\mu$ í $\sigma \gamma o v \tau^{\prime} \dot{\varepsilon} v$

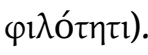

24 Le texte n'est pas très clair sur l'âge auquel Énée sera séparé de ses nourrices. Aphrodite précise d'abord que les Nymphes rendront l'enfant à Anchise au moment où leur fils aura atteint l'âge de l'hêbê, c'est-à-dire à l'adolescence. Mais elle indique ensuite que c'est elle- 
même qui reviendra voir son amant avec l'enfant quand il sera dans sa cinquième année. Il est possible que deux versions différentes aient été en fait ici mises bout à bout (Humbert 1976, n. 2, p. 161). Quoi qu'il en soit, Énée sera un « un jeune être florissant » qui « ressemblera tout à fait à un dieu », façon se souligner sa nature intermédiaire entre mortels et immortels, comme ses nourrices.

Aphrodite poursuit son discours en mettant Anchise en garde :

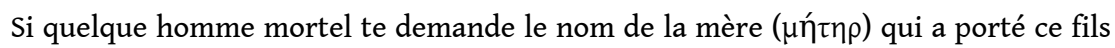
( $\varphi$ í̉ov viòv) sous sa ceinture, n'oublie pas de lui raconter, comme je te l'ordonne : "On dit qu'il est l'enfant (Ěkyovov) d'une Nymphe fraîche comme un bouton de

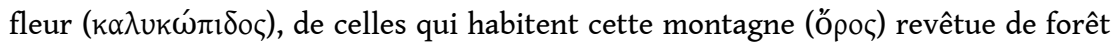
(ט̌ $\lambda \eta ̣) »$. Mais si tu révèles tout, et te vantes follement de t'être uni d'amour à

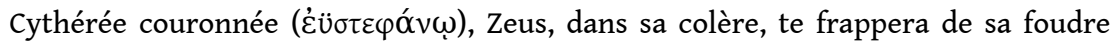
fumante. Tu as tout entendu : songe bien à garder le secret en toi-même, sans me nommer ; redoute plutôt la colère divine (Hymne Homérique à Aphrodite I, 281-290).

Aphrodite, après avoir menti à Anchise sur son identité quand elle se présentait à lui comme une numphê humaine destinée à devenir son épouse, lui ordonne de mentir à son tour en prétendant que l'enfant est né d'une Nymphe.

Le mot de mère ( $\mu$ ń $п \eta \rho)$ apparaît ici explicitement, de même que le lien affectif avec l'enfant ( $\varphi$ ílov viòv). Il ne s'agit donc pas seulement d'une maternité uniquement biologique (malgré l'emploi du terme čkүovov).

La beauté de la prétendue mère d'Énée est particulièrement soulignée, puisqu'elle a la fraîcheur d'un bouton de fleur $(\kappa \alpha \lambda \nu \kappa \omega ́ \pi l \delta \circ \varsigma)$, peut-être de la rose qui était consacrée à Aphrodite (Pirenne-Delforge 1994, p. 412). On ne saurait mieux souligner le parallèle entre Aphrodite et les Nymphes. Au reste, toute l'histoire de l'Hymne homérique à Aphrodite suit le schéma bien connu depuis Homère des amours d'une Nymphe et d'un berger (Larson 2001, p. 81-84).

29 Cette union se révèle terrifiante pour Anchise, quand il apprend la véritable identité de son amante (Hymne homérique à Aphrodite I, 185-190). N'oublions pas que nous sommes en Asie, non loin de la Phrygie (dont Aphrodite avait prétendu être princesse); Anchise craint probablement de subir un sort semblable à celui d'Attis, l'aimé de la grande Mère Cybèle. Le rapprochement d'Aphrodite avec la grande Mère anatolienne ou d'autres divinités orientales (et d'Anchise avec Attis, mais aussi Tammouz, Dumuzi ou Adonis) a été très en faveur dans la première moitié $\mathrm{du} \mathrm{xx}^{\mathrm{e}}$ siècle, mais n'est plus guère accepté aujourd'hui, où la déesse est plutôt rattachée au schéma folklorique des amours d'une Nymphe et d'un berger ${ }^{15}$. Il y a cependant chez Anchise, clairement manifestée, la peur du mâle à la merci d'une entité féminine plus puissante. Ce renversement des rôles traditionnels de genres ne peut que susciter une anxiété qui n'existe pas dans les relations entre dieux masculins et mortelles ${ }^{16}$. Anchise, s'il ébruite son aventure, encourt la colère des dieux et court le risque de mourir foudroyé par Zeus comme le malheureux Iasion qui, dans l'odyssée, avait osé s'unir à la déesse Déméter (Homère, Odyssée, V, 125-128). Tout se passe comme si l'union avec une Nymphe de l'Ida était beaucoup moins dangereuse. Les Nymphes, en plus d'être les nourrices d'Énée, fournissent ainsi un alibi plausible pour expliquer sa naissance. La collectivité des Nymphes fonctionne ici comme un stock inépuisable grâce auquel on peut construire des généalogies, comme dans plusieurs récits de fondation de cités. Vraies nourrices, elles sont cependant de fausses mères. Leur maternité n'est évoquée qu'à travers le mensonge qu'Aphrodite demande à Anchise de raconter pour justifier la naissance de son fils. Les Nymphes apparaissent 
comme des divinités secondaires au service de la déesse de la mixis érotique, dont elles élèvent le fils, tout en fournissant un alibi à Anchise pour cacher l'identité de la véritable mère. Le thème de la dissimulation est donc présent là aussi, mais de façon un peu différente ; il concerne le discours et non une action concrète comme celle de cacher un objet ou une personne dans une grotte, par exemple.

Désirables et sexuellement actives, les Nymphes sont liées ici à la fois aux arbres, aux montagnes et aux grottes. Mortelles, elles apparaissent comme des puissances bienveillantes, de statut intermédiaire entre les dieux et les hommes ${ }^{17}$. Elles se nourrissent d'ambroisie, comme les dieux, même s'il n'est pas dit clairement qu'elles donnent cet aliment divin au jeune héros. L'ambroisie occupe cependant une place singulière dans les histoires de nourrices et d'enfants divins ou héroïques que l'on trouve dans les hymnes homériques. Il suffit de rappeler la grotte pleine d'ambroisie de l'ambrosienne Maïa (Hymne homérique à Hermès I, 230 et 248), et l'Apollon nourri d'ambroisie et de nectar par Thémis peu après sa naissance dans l'Hymne homérique à Apollon I (123-129). Dans l'Hymne homérique à Déméter I (236-237), la déesse, déguisée en vieille femme et engagée pour devenir la nourrice du petit Démophon, le frotte d'ambroisie avant de souffler sur lui et de le cacher dans le feu pour le rendre immortel (Pirenne-Delforge 2010, p. 687-691).

31 Ainsi, dans l'Hymne homérique à Aphrodite, les Nymphes sont de véritables nourrices et de fausses mères. Elles sont, en tout cas, pleines de sollicitude, comme les nourrices de Dionysos dans le deuxième hymne homérique, difficilement datable ${ }^{18}$, qui lui est consacré.

\title{
L'Hymne homérique à Dionysos : les nourrices du dieu
}

\begin{abstract}
Je chante le bruyant Dionysos aux cheveux ceints de lierre, le noble fils (vióv) de Zeus et de la glorieuse Sémélé, celui que les Nymphes à la belle chevelure (†̉úkopor

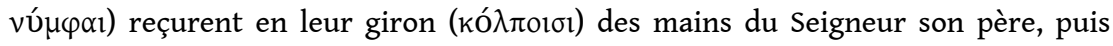

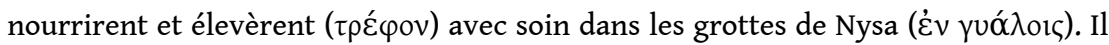
grandit dans un antre odorant, selon le désir de son père, mais en comptant parmi les Immortels. Quand les déesses ( $\theta \varepsilon \alpha \grave{)}$ ) eurent élevé ("$\theta \rho \varepsilon \psi \alpha v)$ celui qui devait être

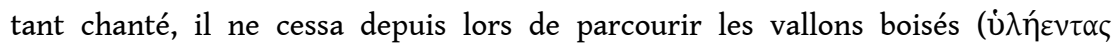
$\dot{\varepsilon} v \alpha u ́ \lambda o u \varsigma)$, tout couronné de lierre et de laurier; les Nymphes le suivaient, et il

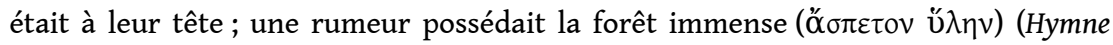
Homérique à Dionysos II, 1-10).
\end{abstract}

Les Nymphes sont présentées ici comme les nourrices de Dionysos. Elles sont identifiées par Mousaios (2 B 18, cité par Hygin, Astronomie, II, 21, 2) et Phérécyde (3 F 90) aux Hyades, filles d'Atlas. Comme leurs sœurs Calypso et Maïa, elles possèdent une «belle

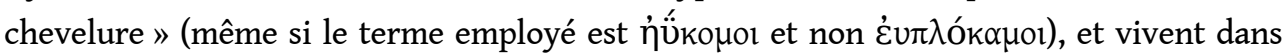
un "antre odorant", à Nysa, qui n'a rien à envier aux grottes-refuges d'Ogygie et du Cyllène. Leur rôle de nourrices rappelle celui des Nymphes des arbres auprès du demidieu Énée dans l'Hymne homérique à Aphrodite. Bien que les nourrices (tithênai) de Dionysos à Nysa soient déjà mentionnées dans l'Iliade (VI, 132-135), c'est la première fois dans les sources épiques conservées qu'elles apparaissent explicitement comme des Nymphes. On remarque que Dionysos, comme Énée, est issu d'une union entre une divinité et un être humain. On retrouve donc là aussi l'affinité des Nymphes avec des êtres qui ont, comme elles, un statut intermédiaire. De plus, Dionysos, bien que «comptant parmi les Immortels ", est l'un des rares dieux de la mythologie grecque à mourir, même si c'est 
dans des traditions orphiques (Detienne 1998, p. 163-207). On pourrait presque faire la même remarque à propos de Zeus en Crète, puisque les habitants de l'île montraient son tombeau (Diodore de Sicile, Bibliothèque historique, VI, 5, 3 ; Cyrille d'Alexandrie, Contre Julien, X, p. 342, D), ce qui les faisait passer aux yeux des autres Grecs pour d'abominables menteurs. Cependant, le Zeus crétois, bien que mortel, est clairement un dieu, sans ascendance humaine comme Dionysos. Le statut des Nymphes ne parait pas non plus poser problème dans l'Hymne à Dionysos, car elles sont clairement qualifiées de « déesses » $(\theta \varepsilon \alpha i)$.

Les nourrices de Dionysos continuent à accompagner le dieu bien longtemps après l'avoir élevé. En effet, quand il est adulte, elles deviennent ses suivantes et parcourent comme lui

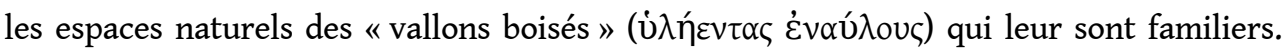
Couronnées de lierre et de laurier, elles restent des puissances liées à la végétation, mais elles apparaissent comme le modèle mythique des Ménades ${ }^{19}$. Leur folie est plus celle de l'extase dionysiaque que de l'inspiration nympholepte. Ce ne sont pas elles qui possèdent, mais elles qui sont possédées. L'association des Nymphes avec Dionysos est ainsi bien attestée sans qu'il soit forcément toujours facile de les distinguer des Ménades, qui peuvent aussi être des femmes mortelles, comme dans Les Bacchantes d'Euripide. Les Ménades, les «Folles ", ont rejeté l'autorité du mâle et abandonné leur sphère normale de l'oikos pour les espaces sauvages de la montagne et des bois (Larson 2001, p. 95) et elles ont un aspect beaucoup plus sauvage et violent que les Nymphes, notamment dans la tragédie d'Euripide où elles pratiquent le diasparagmos et l'omophagie.

Le thème de la dissimulation est un peu moins explicite que dans les autres hymnes, mais il ne faut pas oublier que, selon d'autres textes, Zeus a confié l'enfant Dionysos aux Nymphes de Nysa afin qu'elles l'élèvent dans une grotte lointaine à l'insu d'Héra, son épouse légitime. On serait donc proche de la situation décrite dans l'Hymne homérique à Hermès. Dans l'Hymne homérique à Pan, au contraire, ce n'est pas l'enfant qui est dissimulé, mais la mère qui s'enfuit en rejetant son fils.

\section{La Nymphe rejetant sa maternité : l'Hymne homérique à Pan}

Cet hymne paraît beaucoup plus récent que les précédents; il semble dater en effet du v siècle av. J.-C. ${ }^{20}$ ou même de l'époque hellénistique ${ }^{21}$. L'évocation des Nymphes compagnes de Pan et de sa mère, également qualifiée de Nymphe, peut être rapprochée avec profit de l'Hymne homérique à Hermès, de l'Hymne homérique à Aphrodite et de l'Hymne homérique à Dionysos. Nous laisserons ici de côté les Nymphes compagnes du dieu chèvrepied, mentionnées par deux fois dans le poème (aux vers 3 et 19), pour nous intéresser à la mère de Pan qui abandonne son enfant :

Ils [Pan et les Nymphes] célèbrent les dieux bienheureux et le grand Olympe : par exemple, ils chantaient le bienfaisant Hermès plus que tout autre, en disant comment il est pour tous les dieux le messager rapide, et comment il arriva dans

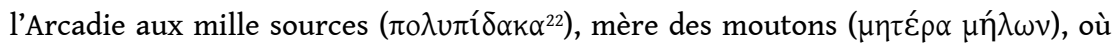
est son domaine du Cyllène. En ces lieux, tout dieu qu'il fût, il gardait chez un homme mortel des moutons à la laine poudreuse : il avait senti venir soudain et grandir dans son cœur le tendre désir de s'unir à une Nymphe aux beaux cheveux,

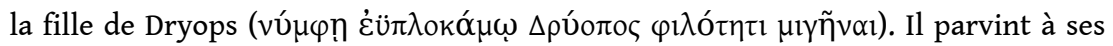

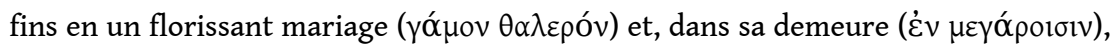
elle donna un fils ( $\varphi$ ì ov vióv) à Hermès; il était en naissant d'aspect monstrueux, 
chèvre-pieds à deux cornes, bruyant et souriant. Elle s'enfuit d'un bond, celle qui devait le nourrir ( $\left.\imath_{\imath} \theta \eta \dot{v \eta}\right)$; et, prise de peur en voyant ce visage farouche et barbu, elle abandonna son enfant $(\pi \alpha \tilde{i} \delta \alpha)$. Le bienfaisant Hermès le prit aussitôt dans ses bras - ce dieu était joyeux au fond de son cœur (Hymne Homérique à Pan, 27-41). chêne ou une mortelle « jeune fille en âge de se marier $^{23}$ ", fille ou fiancée ${ }^{24}$ (numphê) d'un Dryops ancêtre éponyme des Dryopes, les «hommes à visage de chêne " (Pausanias, Description de la Grèce, IV, 34, 9), ou même, selon Lycophron (Alexandra, 480), des Arcadiens eux-mêmes, mangeurs de glands et plus anciens que la lune. Dryops est fils de Lykaon et de Dia selon certains, fils d'Apollon et de la fille de Lykaon (appelée aussi Dia) selon d'autres (Scholie à Apollonios de Rhodes, Argonautiques, I, 1219d; Tzetzès à Lycophron, Alexandra, 480 ; Etymologicum Magnum, s.v. $\Delta \rho v ́ o \psi)$. Il se rattache donc clairement aux premiers rois autochtones d'Arcadie, à la race de Pélasgos, Lykaon et Callisto. Cette dernière est parfois donnée pour la mère de Pan, qu'elle aurait eu de Zeus comme Arcas (Eschyle, fr. 65 b-c Mette = scholie à Euripide, Rhésos, 36). Selon d'autres versions, la mère du dieu chèvre-pied est l'une des nourrices crétoises de Zeus, Aïx (la Chèvre), plus tard assimilée à Amalthée (Épiménide, Fr. 24 Diels-Kranz = Ps.-Ératosthène, Catastérismes, I, 27 = $487 \mathrm{~F} 18 \mathrm{FGrH}$ ), ou l'une des nourrices arcadiennes du roi des dieux, Oinoè (Pausanias, Description de la Grèce, VIII, 47, 3), qui l'aurait eu d'Aither (Ariaithos, 316 F 4, FGrH = Scholie à Euripide, Rhésos, 36). Pour Hérodote (II, 145) et Platon (Cratyle, 408b), il est né de Pénélope et d'Hermès ${ }^{25}$. Ainsi, d'après toutes les versions, sa mère est une Nymphe (n'oublions pas en effet que Pénélope est qualifiée deux fois de numphê dans l'Odyssée, IV, 743 ; XI, 447), et très souvent une nourrice. Pan n'est donc pas seulement le compagnon de ces puissances, mais aussi le fils de l'une d'entre elles.

Dans l'Hymne, l'union entre Hermès et la fille ou fiancée de Dryops semble s'effectuer dans la tendresse du sentiment amoureux ( $\left.\varphi \imath \lambda o ́ \tau \eta \tau \imath \mu \imath \tilde{\eta} v \alpha l^{26}\right)$, et rappelle l'union des Nymphes et d'Hermès dans l'Hymne homérique à Aphrodite. Cette précision permet de souligner le charme irrésistible de la fille de Dryops, caractéristique éminemment nymphique. Son union avec le dieu est même qualifiée de "florissant mariage » (үó́uov $\theta \alpha \lambda \varepsilon \rho o ́ v)$, ce qui semble la faire passer du statut de numphê à celui de gunê. Ce passage est aussi symbolisé par le transfert des espaces pastoraux (où a lieu l'union sexuelle avec le

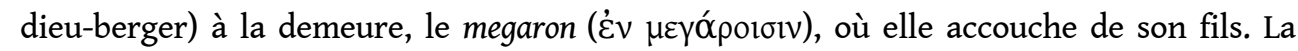
qualification de ce dernier comme pỉ̀ov vióv renvoie plus à la sollicitude du père, Hermès, qu'à celle de la mère, la Nymphe.

contraste entre la beauté de la Nymphe et la laideur de Pan est accentué par l'effroi que suscite l'aspect thériomorphe de son rejeton chez la mère, qui s'enfuit d'un bond et renonce à le nourrir. 
41 À la mère, nourrice ( $₫ 1 \theta \eta ́ v \eta)$, qui prend ses jambes à son cou correspond un autre départ : Hermès recueille le nourrisson, l'enveloppe dans la peau d'un lièvre des montagnes et s'empresse de gagner avec lui l'olympe, où les dieux, à la différence de la mère, sont charmés par le nouveau-né. Mais l'évocation de Dionysos comme étant le plus charmé par Pan laisse entendre que l'on est dans l'inversion des valeurs humaines. Selon Philippe Borgeaud (1979, p.155-156), ce double mouvement de retrait (épouvante humaine, éloignement du dieu vers l'olympe) éclaire le sens de la panique : «le désordre instauré par celle-ci répond à un éloignement exagéré du divin par rapport aux hommes, à une rupture qui a pour résultat que l'homme perd pied, quitte le réel, livré à ses fantasmes par le dieu». Cette panique ne saurait être provoquée par les Nymphes, dont la possession, la nympholepsie, paraît bien différente de la panolepsie, la possession par Pan (Borgeaud p. 163-166).

42 Le qualificatif de «mère " n'est pas appliqué à la fille de Dryops. Dans le poème, seule

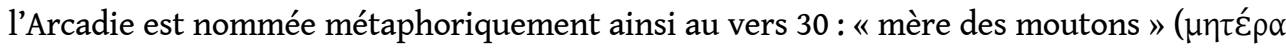

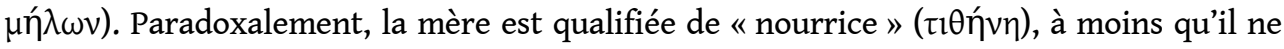
faille voir ici deux personnages féminins distincts - la mère et la nourrice -, comme le suggère Oliver Thomas (2011, p. 162). En tout cas, les deux s'enfuient, effrayées par la laideur du nouveau-né. La Nymphe ne remplit pas ses obligations de sollicitude maternelle, elle n'est plus qu'une simple génitrice. La trophê est assurée par le père, Hermès, qui emmène l'enfant sur l'olympe pour le présenter aux autres dieux qui s'en émerveillent. La mère se cache, et l'on retrouve encore ici le thème de la dissimulation, sous une autre forme, plus négative...

\section{Conclusion}

Pour conclure, on peut noter une certaine polyvalence des Nymphes par rapport aux fonctions maternelle et courotrophe dans les Hymnes homériques. Dans l'Hymne homérique à Hermès, Maïa - dont le nom renvoie à l'une des appellations pour la nourrice en grec - est à la fois une mère et une nourrice, cumulant maternité biologique et sociale. Dans l'Hymne homérique à Aphrodite, on trouve la première occurrence des Nymphes nourrices; mais l'une des Nymphes de l'Ida est censée assumer la maternité d'Énée, le rôle d'Aphrodite devant rester dissimulé. Dans l'Hymne homérique à Dionysos, on retrouve cette fonction courotrophe, mais les Nymphes continuent à suivre le dieu après l'avoir élevé, et participent à son thiase. Enfin, l'Hymne homérique à Pan offre l'exemple d'une maternité biologique sans maternité sociale, la jeune mère rejetant son enfant.

Malgré la variété de ce rapport aux rôles de mère et de nourrice, on note néanmoins une certaine constante dans le mode d'action des Nymphes. Les soins donnés à l'enfant se font de manière cachée, en secret, souvent dans l'obscurité d'une grotte, afin de dissimuler un enfant illégitime à sa marâtre (Hermès et Dionysos). Dans l'Hymne homérique à Aphrodite, les Nymphes, en plus de leur fonction nourricière, servent à dissimuler la véritable mère, tandis que cette dernière s'enfuit et disparaît dans l'Hymne homérique à Pan.

Cette sollicitude dans la dissimulation se retrouve dans d'autres sources que les Hymnes homériques. Ainsi, chez Callimaque, dans l'Hymne à Zeus (28-54), la Nymphe Néda, après avoir assisté Rhéia lors de son accouchement secret en Arcadie, à l'insu de Cronos, transporte le bébé Zeus en Crète afin qu'il y soit élevé par les Nymphes Méliai ${ }^{27}$. On retrouve l'histoire de l'enfance de Zeus en Crète dans la Bibliothèque du Pseudo-Apollodore 
(I, 5). Les Nymphes s'occupent d'un enfant menacé, cette fois-ci par son père. Ce n'est peut-être pas un hasard si les Nymphes veillent sur les jeunes années des seuls dieux grecs qui ont une enfance, Zeus, Hermès et Dionysos (Rudhardt 2006), probablement parce que leur statut est incertain: Zeus risque d'être englouti par Cronos s'il est découvert; Hermès ne participe pas encore aux timai des Olympiens au début du récit de l'hymne qui lui est consacré (Leduc 2005 ; Jaillard 2007) ; Dionysos, fils d'une mortelle née elle-même d'un mortel et d'une immortelle, se situe à la limite de la mortalité et de l'immortalité. Les Nymphes ont elles aussi un statut intermédiaire entre les dieux et les hommes, notamment dans l'Hymne homérique à Aphrodite. Leur fonction courotrophe semble en faire des puissances médiatrices protégeant le passage entre deux statuts, de l'enfance à l'âge adulte. Leur sollicitude dans la dissimulation se retrouve dans d'autres domaines. Ainsi, pour ne donner que le seul exemple de l'Odyssée, Calypso cache Ulysse pendant sept ans tout en prenant soin de lui, tandis que ce dernier, à son arrivée à Ithaque, cache le trésor que lui ont offert les Phéaciens dans la grotte des Nymphes Naïades. Il n'y a donc pas que dans les domaines de la maternité et du soin des enfants que les Nymphes opèrent des dissimulations, que l'on s'essaiera à dévoiler peu à peu...

\section{BIBLIOGRAPHIE}

Acker C. (2002), Dionysos en transe : la voix des femmes, Paris.

Ando V. (1996), « Nymphe : la sposa e le ninfe », QUCC 52-1, p. 47-79.

Ballabriga A. (1986), Le Soleil et le Tartare : l'image mythique du monde en Grèce archaïque, Paris.

Ballentine F. G. (1904) « Some Phases of the Cult of the Nymphs », HSPh 15, p. 77-119.

Borgeaud Ph. (1979), Recherches sur le dieu Pan, Genève.

Brillet-Dubois P. (2001), « Les liaisons dangereuses. Dieux et mortels dans l'Hymne homérique à Aphrodite ", Homère, Europe 865, p. 250-260.

Bruit Zaidman L. (2013), « Déméter-Mère et les figures de la maternité », Mètis N.S. 11, p. 93-108.

Buxton R. (1996), La Grèce de l'imaginaire. Les contextes de la mythologie, Paris.

Calame Cl. (1994-1995), « Les Hymnes homériques. Modalités énonciatives et fonctions », Mètis IX-X, p. 391-400.

- (1995), «Variations énonciatives, relations avec les dieux et fonctions poétiques dans les Hymnes homériques ", MH 52, p. 2-19, repris dans Id. (2005), Masques d'autorité. Fiction et pragmatique dans la poésie grecque antique, Paris, p. 43-71.

- (1996), L'Éros dans la Grèce antique, Paris.

- (2011), « The Homeric Hymns as poetic offerings: musical and ritual relationships with the gods », dans Faulkner A. éd., The Homeric Hymns. Interpretative Essays, Oxford, p. 334-358.

- (2012), «Les Hymnes homériques comme prières poétiques et comme offrandes musicales. Le chant hymnique en acte », Mètis N.S. 10, p. 51-76. 
Chantraine P. (1999), Dictionnaire étymologique de la langue grecque : histoire des mots, Paris, p. 758-759 (s. v. vú $\varphi \varphi \eta)$.

Clay J. S. (1989), The Politics of Olympus: Form and Meaning in the Major Homeric Hymns, Princeton.

Clay J. (2011), « The Homeric Hymns as genre », dans Faulkner A. éd., The Homeric Hymns. Interpretative essays, Oxford, p. 232-253.

Cova A. (2005), « Où en est l'histoire de la maternité ? », Clio. Histoire, Femmes et Sociétés 21, p. 189-211.

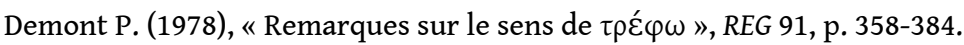

Detienne M. (1998), Dionysos mis à mort, $2^{\mathrm{e}}$ éd., Paris.

Evelyn White H. G. (1967), Hesiod, the Homeric Hymns and Homerica, Londres.

Faulkner A. (2008), The Homeric Hymn to Aphrodite: Introduction, Text and Commentary, Oxford.

- éd. (2011), The Homeric Hymns. Interpretative Essays, Oxford.

Gherchanoc F. et Bonnard J.-B. (2013), « Mères et maternités en Grèce ancienne. Quelques éléments historiographiques et pistes de réflexion », Mètis N.S. 11, p. 7-27.

Hadzistelou-Price Th. (1978), Kourotrophos. Cults and Representations of the Greek Nursing Deities, Leyde.

Humbert J. (1976), Homère, Hymnes, Paris, 1976.

Jaillard D. (2007), Configurations d'Hermès : une « théogonie hermaïque », Kernos Suppl. 17, Liège.

Kahn L. (1978), Hermès passe : ou les ambiguittés de la communication, Paris.

Knibiehler Y. (2008), « Préface », dans Dermenjian G., Guilhaumou J. et Lapied M. éd., La puissance maternelle en Méditerranée. Mythes et représentations, Arles, p. 11-18.

Larson J. (2001), Greek Nymphs: Myth, Cult, Lore, Oxford.

Leduc Cl. (2005), « "Le pseudo-sacrifice d'Hermès” Hymne homérique à Hermès I, vers 112-142. Poésie rituelle, théologie et histoire ", Kernos 18, p. 141-165.

Lefkowitz M. R. (2002), « Predatory goddesses », Hesperia 71, p. 325-344.

Loraux N. (1991), « Qu'est-ce qu'une déesse ? » dans Schmitt Pantel P. éd., Histoire des femmes en Occident. 1. L'Antiquité, Paris, p. 31-62.

Mactoux M.-M. (1975), Pénélope : légende et mythe, Paris.

Nagy G. (2011), « The earliest phases in the reception of the Homeric Hymns », dans Faulkner A. éd., The Homeric Hymns. Interpretative Essays, Oxford, p. 280-333.

Pache C. O. (2011), A Moment's Ornament. The Poetics of Nympholepsy in Ancient Greece, OxfordNew York.

Pirenne-Delforge V. (1994), L'Aphrodite grecque, Kernos Suppl. 4, Liège.

- (2004), « Qui est la Kourotrophos athénienne? ", dans Dasen V. éd., Naissance et petite enfance dans l'Antiquité, Fribourg-Göttingen, p. 171-185.

- (2005), « La maternité des déesses grecques et les déesses-mères : entre mythe, rite et fantasme ", Clio. Histoire, Femmes et Sociétés 21, p. 129-138. 
- (2008), « Maternité et divinité en Grèce antique : l'exemple de Déméter », dans Dermenjian G., Guilhaumou J. et Lapied M. éd., La puissance maternelle en Méditerranée. Mythes et représentations, Arles, p. 37-54.

- (2010), « Nourricières d'immortalité : Déméter, Héra et autres déesses en pays grec », Paedagogica Historica 46/6, p. 685-697.

Pironti G. (2007), Entre ciel et guerre. Figures d'Aphrodite en Grèce ancienne, Kernos Suppl. 18, Liège. Rudhardt J. (1971), Le thème de l'eau primordiale dans la mythologie grecque, Berne.

- (1990), « De la maternité chez les déesses grecques », RHR 207-4, p. 367-388.

- (2006), «L'enfance des dieux », dans Les dieux, le féminin, le pouvoir : enquêtes d'un historien des religions, Genève, p. 73-93.

Scheid J. (2012), «Des divinités qui meurent. Réflexions sur les nymphes », dans D’Intino S. et Guenzi C. éd., Aux abords de la clairière. Études indiennes et comparées en l'honneur de Charles Malamoud, Turnhout, p. 163-173.

Segal Ch. (1974), « The Homeric Hymn to Aphrodite: a structuralist approach », CW 67, p. 205-219. Sourvinou-Inwood Ch. (2005), Hylas, the Nymphs, Dionysos and Others: Myth, Ritual, Ethnicity, Stockholm.

Stehle E. (1996), « Sappho's gaze: fantasies of a goddess and young man », dans Greene E. éd., Reading Sappho: Contemporary Approaches, Berkeley-Los Angeles, p. 193-225.

Thebaud Fr. (2005), « Éditorial » au dossier Maternités, Clio. Histoire, Femmes et Sociétés 21, p. 9-16.

Thomas O. (2011), « The Homeric Hymn to Pan ", dans Faulkner O. éd., The Homeric Hymns. Interpretative essays, Oxford, p. 151-172.

Vamvouri Ruffy M. (2004), La fabrique du divin : les Hymnes de Callimaque à la lumière des Hymnes homériques et des Hymnes épigraphiques, Kernos Suppl. 14, Liège.

Vernant J.-P. (1974), « Le mythe prométhéen chez Hésiode », dans Mythe et société en Grèce ancienne, Paris, 1974, p. 177-194.

- (1979), «À la table des hommes : mythe de fondation du sacrifice chez Hésiode », dans Detienne M. et Vernant J.-P. éd., La cuisine du sacrifice en pays grec, Paris, p. 37-132.

Villanueva-Puig M.-Ch. (2009), Ménades : recherches sur la genèse iconographique du thiase féminin de Dionysos des origines à la fin de la période archaïque, Paris.

Vilatte S. (1991), « La nourrice grecque : une question d'histoire sociale et religieuse », AC 40, p. 5-28.

Winkler J. J. (2005), Désir et contraintes en Grèce ancienne, Paris.

\section{NOTES}

1. On laisse ici de côté deux autres significations du terme "maternité » en français, qui ne concernent pas la période antique: le lieu (l'hôpital) où s'effectuent les accouchements; une œuvre d'art représentant la mère et l'enfant. Pour un bilan historiographique sur les mères et la maternité en Grèce ancienne, voir Gherchanoc et Bonnard 2013.

2. Sur la maternité de Déméter, voir Pirenne-Delforge 2008 et Bruit Zaidman 2013.

3. Sur la maternité des déesses, voir aussi Rudhardt 1990 et Loraux 1991, p. 48-56. 
4. Comme dans la première partie de l'article de Vinciane Pirenne-Delforge (2010) qui étudie les figures divines "nourricières d'immortalité ", en se limitant aux quatre grands hymnes à Apollon, Hermès, Déméter et Aphrodite.

5. Sur les hymnes homériques, parmi une abondante bibliographie, voir notamment Clay 1989 et, sur leur dimension performative, Calame 1994-1995, Calame 1995, Faulkner 2011, Clay 2011, Nagy 2011, Calame 2011, Calame 2012.

6. Faulkner 2011, p. 13 le date de la fin du vi ${ }^{\mathrm{e}}$ siècle, voire du début du $\mathrm{v}^{\mathrm{e}}$ siècle av. J.-C.

7. Nous citons les hymnes dans la traduction de Humbert 1967.

8. Ce n'est pas le cas de toutes les grottes mythiques, n'en déplaise à la psychanalyse jungienne !

9. Hésiode, Théogonie, 536-541. Le poète utilise le verbe k $\alpha \lambda u ́ \psi \alpha c$. Sur le sacrifice de Prométhée, voir Vernant 1974 ; Detienne et Vernant 1979, p. 37-132.

10. L'ambroisie semble trouver ses origines dans l'eau primordiale d'Okéanos ; cf. Rudhardt 1971, p. 94-97.

11. Humbert 1967, p. 146 ; Evelyn White 1967, p. xxxviii ; Faulkner 2008, p. 14 penche même pour le milieu du VII ${ }^{\mathrm{e}}$ siècle, sans exclure l'idée d'une datation plus haute (fin du viII ${ }^{\mathrm{e}}$ siècle).

12. On n'a pas de Nymphes nourrices chez Homère, ni chez Hésiode (chez qui les Océanides qui prennent soin des jeunes gens ne sont pas qualifiées de Nymphes et s'occupent, en outre, d'adolescents plutôt que de nourrissons...).

13. Sur la mortalité des Nymphes, voir dernièrement Scheid 2012.

14. Ganymède est enlevé par Zeus en raison de sa beauté, Tithon est enlevé par Eos amoureuse de lui, les Nymphes de l'Ida s'unissent à Hermès dans des grottes et, enfin, Anchise s'unit à Aphrodite.

15. Larson 2001, p. 82 ; le rapprochement avec des motifs proche-orientaux occupe encore une place non négligeable chez Faulkner 2008, p. 18-22.

16. Larson 2001, p. 83 ; sur les unions de déesses avec des hommes mortels, voir aussi Stehle 1996 ; Lefkowitz 2002 ; Brillet-Dubois 2001 ; Pache 2011 (qui a une conception très extensive du phénomène de "nympholepsie ", englobant selon elle les enlèvements de jeunes mortels par des Nymphes, mais aussi par des déesses qu'elle qualifie de «nympholeptiques », par ex. p. 13).

17. Sur cette ambivalence du statut des Nymphes, voir par exemple Faulkner 2008, p. 185-186.

18. Humbert 1976, p.170. Selon Faulkner 2011, p.15, il peut être seulement daté, comme la plupart des hymnes courts, du VII ${ }^{\mathrm{e}}$ ou du vi ${ }^{\mathrm{e}}$ siècle av. J.-C.

19. Sur le rapport des Nymphes et des Ménades (et la distinction entre les deux groupes, notamment dans l'iconographie), cf. Acker 2002, p. 61-71 et Villanueva-Puig 2009, p. 85-88.

20. Faulkner 2011, p. 15 ; Thomas 2011, p. 169 ; pour eux, le poème n'est pas antérieur à 500 av. J.C., moment où le culte de Pan se diffuse hors d'Arcadie, notamment en Attique après la bataille de Marathon en 490 av. J.-C., suivant le témoignage d'Hérodote (VI, 105).

21. Pour Jean Humbert (1976), il pourrait être l'œuvre d'un poète de la génération de Callimaque et de Théocrite.

22. Dans l'Hymne homérique à Aphrodite I, 54, le même terme qualifie les hauteurs montagneuses de l'Ida où Anchise garde ses bœufs.

23. Sur le double sens du mot numphê en grec, voir par exemple Chantraine 1999, p. 758-759 (s. v. vú $\mu \varphi \eta)$; Calame 1996, p. 140-145 ; Ando 1996, p. 47-79.

24. Thomas 2011, p. 151-172, traduit numphê par « wife» (p. 167) ou « bride» (p. 166, 167 et 169). 25. Sur Pénélope mère de Pan, voir Mactoux 1975, p. 221-230. Les différentes sources donnent Hermès, mais aussi Apollon, les Prétendants ou Ulysse comme pères de Pan.

26. La philotês apparaît cependant comme une puissance ambiguë, fille de Nuit selon Hésiode ( Théogonie, 224), et que Gabriella Pironti (2007, p. 40) propose de traduire de manière neutre par « relation, union intime » dans un contexte érotique.

27. Sur le rapport des hymnes de Callimaque avec les hymnes homériques, voir Vamvouri Ruffy 2004. 


\section{RÉSUMÉS}

L'étude de quatre hymnes homériques laisse apparaître une certaine polyvalence des Nymphes par rapport aux fonctions maternelle et courotrophe. Dans l'Hymne homérique à Hermès, Maïa est à la fois une mère et une nourrice, cumulant maternité biologique et sociale. Dans l'Hymne homérique à Aphrodite, on trouve la première occurrence des Nymphes nourrices ; mais l'une des Nymphes de l'Ida est censée assumer la maternité d'Énée, le rôle d'Aphrodite devant rester dissimulé. Dans l'Hymne homérique à Dionysos, on retrouve cette fonction courotrophe, toutefois les Nymphes continuent à suivre le dieu après l'avoir élevé, et participent à son thiase. Enfin, l' Hymne homérique à Pan offre l'exemple d'une maternité biologique sans maternité sociale, la jeune mère rejetant son enfant. Malgré la variété de ce rapport aux rôles de mère et de nourrice, on note une certaine constance dans le mode d'action des Nymphes, qui semble se caractériser par une forme de sollicitude dans la dissimulation, faisant de ces divinités des puissances médiatrices protégeant le passage entre deux statuts, de l'enfance à l'âge adulte.

The study of four Homeric hymns reveals some versatility of Nymphs with regard to their maternal and courotrophic functions. In the Homeric Hymn to Hermes, Maia is both a mother and a nurse, combining biological and social motherhood. The Homeric Hymn to Aphrodite offers the first occurrence of nursing Nymphs, but one of the Idaean Nymphs is supposed to be Aeneas' mother, as the role of Aphrodite remains concealed. The courotrophic function is also attested in the Homeric Hymn to Dionysus, but Nymphs continue to follow the god after raising him, taking part in his thiasos. Finally, the Homeric Hymn to Pan shows an example of biological motherhood without social motherhood, the young mother rejecting her child. Despite the variety of these different roles of mother and nurse, there is some constancy in the mode of action of the Nymphs, which seems to be characterized by a form of care in hiding, making these deities mediating entities protecting the transition between two statuses, from childhood to adulthood.

\section{INDEX}

Mots-clés : Nymphes (divinités grecques), maternité, courotrophie, nourrice, hymnes homériques

Keywords : Nymphs (greek deities), motherhood, courotrophy, nurse, homeric hymns

\section{AUTEUR}

\section{SÉBASTIEN DALMON}

Bibliothèque interuniversitaire de la Sorbonne, CADIST Antiquité ; ANHIMA - UMR8210 